\title{
The acute care of the elderly unit: providing rapid specialised care for frail older people
}

\author{
Authors: Towhid Imam, Robert Goldsby-West, Nima Hashemi, Alan Earnshaw, Joanne Bramble, Joe Rehman \\ and Wallace Tan
}

\begin{abstract}
Aims
The aim of the acute care of the elderly unit was to deliver early comprehensive geriatric assessment providing robust discharge plans resulting in more efficient and safer care.
\end{abstract}

\section{Methods}

A short-stay inpatient facility with daily consultant ward rounds of 14 beds was created. A multidisciplinary approach was integral involving doctors, therapists, pharmacy and social worker support, old age psychiatry and the voluntary sector. A general practitioner / emergency department hotline to a consultant geriatrician with rapid access outpatient clinic was available. Appropriate patients were also chosen for admission from the emergency and acute medical services to relieve pressure on the front door. The unit had close communication with step up rehabilitation beds in the community. The Cerner electronic health record system was used to measure the impact of the unit.

\section{Results}

Approximately 100 patients were seen per month over a 1-year period. The impact was a $10 \%$ increase in the percentage of patients discharged within 24 hours of admission and a $15 \%$ increase within 72 hours of admission. There was reduced bed occupancy with a reduction in the average length of stay in the hospital of 5 days for patients aged 80 and over. A reduction in the re-admission numbers for patients aged 70 and above was observed. This enabled 80 inpatient beds to be closed over a 3-year period. The most common diagnoses were urinary tract infection, falls, respiratory tract infection, syncope, cellulitis and constipation.

\section{Conclusion}

The acute care of the elderly unit has improved the efficiency and delivery of safer care to the older population of Croydon. Discharges were more streamlined for several reasons. Social care provision was efficient as sections for care provision were not required. Pharmacists participated in ward rounds to ensure medications were promptly arranged and problems solved swiftly. Patients admitted with a fall were seen 17 hours quicker by a physiotherapist. The unit also streamlined referrals to community services improving the care of the discharged patient and preventing readmission. Challenges faced included inappropriate use of the unit during busy periods and the need for better resources downstream in the community. Making frailty everyone's business with a shared vision across the emergency department, clinical commissioning group (CCG) and acute physicians was key to the success of the unit.

\section{Conflict of interest statement}

None declared. 